\title{
BMJ Open Relationship between classic vascular risk factors and cumulative recurrent cardiovascular event burden in patients with clinically manifest vascular disease: results from the UCC-SMART prospective cohort study
}

Tamar Irene de Vries, ${ }^{1}$ Jan Westerink, ${ }^{2}$ Michiel L Bots, ${ }^{3}$ Folkert W Asselbergs, ${ }^{4,5}$ Yvo M Smulders (1) , ${ }^{6}$ Frank L J Visseren (1) ${ }^{7}$

To cite: de Vries TI, Westerink J, Bots ML, et al. Relationship between classic vascular risk factors and cumulative recurrent cardiovascular event burden in patients with clinically manifest vascular disease: results from the UCC-SMART prospective cohort study. BMJ Open 2021;11:e038881. doi:10.1136/ bmjopen-2020-038881

- Prepublication history for this paper is available online. To view these files, please visit the journal online (http://dx.doi org/10.1136/bmjopen-2020038881).

Received 28 May 2020 Revised 19 August 2020 Accepted 09 0ctober 2020
Check for updates

(c) Author(s) (or their employer(s)) 2021. Re-use permitted under CC BY-NC. No commercial re-use. See rights and permissions. Published by BMJ.

For numbered affiliations see end of article.

Correspondence to Dr Frank L J Visseren; F.L.J.Visseren@umcutrecht.nl

\section{ABSTRACT}

Objective The aim of the current study was to assess the relationship between classic cardiovascular risk factors and risk of not only the first recurrent atherosclerotic cardiovascular event, but also the total number of nonfatal and fatal cardiovascular events in patients with recently clinically manifest cardiovascular disease (CVD). Design Prospective cohort study.

Setting Tertiary care centre.

Participants 7239 patients with a recent first manifestation of CVD from the prospective UCC-SMART (Utrecht Cardiovascular Cohort - Second Manifestations of ARTerial disease) cohort study.

Outcome measures Total cardiovascular events, including myocardial infarction, stroke, vascular interventions, major limb events and cardiovascular mortality.

Results During a median follow-up of 8.9 years, 1412 patients had one recurrent cardiovascular event, while 1290 patients had two or more recurrent events, with a total of 5457 cardiovascular events during follow-up. The HRs for the first recurrent event and cumulative event burden using Prentice-Williams-Peterson models, respectively, were $1.36(95 \% \mathrm{Cl} 1.25$ to 1.48$)$ and 1.26 (95\% Cl 1.17 to 1.35$)$ for smoking, 1.14 (95\% Cl 1.11 to 1.18) and 1.09 (95\% Cl 1.06 to 1.12) for non-high-density lipoprotein (HDL) cholesterol, and $1.05(95 \% \mathrm{Cl} 1.03$ to 1.07 ) and 1.04 (95\% Cl 1.03 to 1.06) for systolic blood pressure per $10 \mathrm{~mm} \mathrm{Hg}$.

Conclusions In a cohort of patients with established CVD, systolic blood pressure, non-HDL cholesterol and current smoking are important risk factors for not only the first, but also subsequent recurrent events during follow-up. Recurrent event analysis captures the full cumulative burden of CVD in patients.

\section{INTRODUCTION}

In cardiovascular (CV) clinical trials investigating treatment effects and in cohort studies, there is a focus on the first CV event

\section{Strengths and limitations of this study}

- This study uses a total (cumulative) event analysis to better capture the entire clinical disease burden than time-to-first event alone.

- The study has a prospective design with systematic collection of both baseline variables and outcomes to minimise bias.

- Several sensitivity analyses were performed to ensure robustness and validity of the results.

- All outcomes are weighed equally in the total event analysis, while in clinical practice not all endpoints have equal clinical relevance.

- Only baseline measurements were studied, meaning that changes in risk factors were not taken into account over longer follow-up.

during follow-up. In clinical practice not only the first (recurrent) CV event is relevant for healthcare professionals and patients, but all CV events during the next years or even during a patient's lifespan.

Evaluating only the first (recurrent) CV event during follow-up does not use the complete clinical information available, as subjects with a first non-fatal event can continue to be followed during a study period and can experience numerous additional events during the course of follow-up. Ignoring these additional events may therefore not reflect the total cardiovascular disease (CVD) burden of an individual patient over time. ${ }^{1}$ Moreover, it is likely that the relation between a risk factor and an endpoint may be different for the first event compared with the total disease burden. ${ }^{2}$ This may be especially true for modifiable risk factors, which may diminish in importance after the initiation of 
secondary prevention. Consequently, time-to-first event analyses may not capture the full clinical impact of a risk factor. Clinically, therefore, it is also of interest to study the effect of risk factors or treatment on the cumulative event burden. The commonly used composite endpoint, including only 'hard' endpoints such as myocardial infarction, stroke and CV death, may also not capture the clinical burden for a patient, as revascularisation procedures and peripheral artery disease are also relevant events or outcomes in clinical practice in terms of loss of quality of life and economic costs. ${ }^{3}$ Investigating the relationship between risk factors and the total event burden may therefore provide additional information compared with first events in a composite endpoint alone. Several randomised clinical trials studying risk factor reduction in $\mathrm{CV}$ research have published results of total event analyses in the last decade, which show that recurrent event analyses are a better measure of the total disease burden..$^{3-8}$ However, few total (or cumulative) event analyses have previously been published that study the relation between classic vascular risk factors and CV outcomes in an observational cohort study. ${ }^{910}$

Patients with established CVD are known to be at higher risk of future (recurrent) events compared with patients without CVD. ${ }^{11}$ The aim of the current study is to assess the relationship between classic modifiable $\mathrm{CV}$ risk factors blood pressure (BP), cholesterol and smoking and the risk of not only the first recurrent atherosclerotic CV event, but also the total number of recurrent $\mathrm{CV}$ events in patients with a recent first manifestation of CVD.

\section{METHODS}

\section{Study population}

Data from the Utrecht Cardiovascular Cohort - Second Manifestations of ARTerial disease (UCC-SMART) study were used, an ongoing prospective cohort study at the University Medical Centre Utrecht, The Netherlands. From September 1996 onwards, patients over 18 years old referred to our institution with clinically manifest vascular disease or vascular risk factors were eligible for participation. The rationale and study design of the UCCSMART cohort study have been published previously. ${ }^{12}$ Written informed consent was obtained from all participants. For the current study, data from 7239 participants with a recent first manifestation of CVD enrolled between 1996 and 2018 were used.

\section{Baseline measurements}

After inclusion, study participants underwent a standardised vascular screening consisting of a health questionnaire including medical history and CV risk factors, physical examination and laboratory testing in fasting state. ${ }^{12}$ Smoking was recorded as current versus past/ never smoker. Non-high-density lipoprotein (HDL) cholesterol was defined as total cholesterol minus HDL cholesterol, both measured in fasting state. Systolic blood pressure (SBP) was measured using an automatic oscillometric device on both arms, and the mean of the highest arm was used.

\section{Follow-up}

During follow-up, information on hospitalisation, outpatient clinic visits and (CV) events was obtained biannually through questionnaires. All available data were collected on reported events. All events were independently evaluated by three members of the UCC-SMART cohort endpoint committee. The primary endpoint of the current study consisted of non-fatal atherosclerotic CV events, including myocardial infarction, stroke, major adverse limb events (MALE), revascularisation procedures and other vascular interventions, and death due to CV causes. MALE was defined as any amputation above the forefoot and revascularisation procedures of the lower limbs. Other vascular interventions included coronary revascularisation, procedures of the abdominal aorta, or revascularisation of the carotid arteries, renal arteries or iliac arteries. Death was reported by the general practitioner, treating specialist or relatives.

Duration of follow-up was defined as the period between study inclusion and death, loss to follow-up or the preselected date of 1 March 2018. In total, 561 (6.6\%) participants were lost to follow-up during the study period.

\section{Statistical analysis}

All analyses were conducted with R V.3.5.1 statistical software (www.r-project.org; packages survival, Hmisc, dplyr, MASS, ggplot2). Missing values $(<1 \%)$ were imputed using predictive mean matching (aregImpute algorithm in R, Hmisc package). ${ }^{13}$

Baseline clinical characteristics were presented as frequencies with percentages for categorical variables, and mean with SD for continuous variables.

Non-parametric mean cumulative function curves were created for total CV events using the Nelson-Aalen estimator. ${ }^{14}$ The mean cumulative function represents the expected (ie, mean) cumulative number of events for a patient at a given point in time after inclusion. For comparative purposes, Kaplan-Meier curves were also created for the first CV event and plotted with the mean cumulative function curves. To visualise the effect of the risk factors of interest (current smoking, non-HDL cholesterol and SBP), plots were also created stratified for the presence of the investigated risk factors: current smoking vs no current smoking; SBP $\geq 140 \mathrm{~mm} \mathrm{Hg}$ vs $<140$ $\mathrm{mm} \mathrm{Hg}$; and non-HDL cholesterol $\geq 2.6 \mathrm{mmol} / \mathrm{L}$ vs $<2.6$ $\mathrm{mmol} / \mathrm{L}$, based on the European Society of Cardiology thresholds. $^{15}$

As the study aim was to determine the aetiological relationship between the modifiable risk factors of interest, all analyses were adjusted for potential confounders based on previous literature, which included, where appropriate, age, sex, alcohol use, type of baseline vascular disease, current smoking, SBP and non-HDL at baseline. The analyses for non-HDL cholesterol were additionally adjusted for statin therapy and diabetes; SBP 
was additionally adjusted for BP-lowering medication and estimated glomerular filtration rate.

Time-to-first event was studied using Cox proportional hazard models. The composite endpoint consisted of all previously described CVD endpoints. Linearity of the relationship between non-HDL cholesterol and SBP with the outcome was assessed with restricted cubic splines. The assumption of proportionality was visually checked by plotting Schoenfeld residuals. Prentice-Williams-Peterson (PWP) analysis, an extension of the Cox proportional hazard model to model recurrent events, was performed to assess the relationship between the risk factors of interest and the total event burden, ${ }^{16}$ as it has been suggested that this is the most robust of the recurrent event modelling methods. ${ }^{2}$ The PWP model is a conditional model, meaning that patients are only part of the risk set for event $k$ when they have experienced event $k-1$.

\section{Sensitivity analyses}

As has been recommended, several sensitivity analyses were performed to demonstrate that an apparent causal effect does not depend on the chosen statistical method. ${ }^{17} 18$ Negative binomial regression is an attractive method for recurrent event modelling because it accommodates heterogeneity among patients. It assumes that each participant has events according to their own individual, specific event rate through a random effect term which varies according to a gamma distribution. ${ }^{19-21} \mathrm{~A}$ negative binomial regression model was therefore used to obtain estimates of the effect of the risk factors of interest on the outcome event rate, including all nonfatal and fatal CV events. A disadvantage of negative binomial regression is that it does not take time to event into account, which may bias the results if there is unequal follow-up..$^{20}$

Additional sensitivity analyses were performed using the Andersen-Gill and Wei-Lin-Weissfeld (WLW) models, ${ }^{22} 23$ both extensions of the Cox proportional hazard model to model recurrent events. In the WLW model, individual patients are included in the risk set for event $k$ regardless of whether they have experienced event $k-1$. Both the PWP and WLW models use follow-up time from inclusion, a 'total time' approach, in contrast to the 'gap time' approach of Andersen-Gill, where the times between consecutive events are modelled rather than the time from baseline. ${ }^{19}$ The Andersen-Gill method assumes that all events are independent. This assumption is clearly violated, as the risks of $\mathrm{CV}$ events within one individual are associated. Therefore, robust SEs may be used with the Andersen-Gill method to account for heterogeneity. ${ }^{24}$ All recurrent event analyses were limited to the first five events.

To investigate the role of competing events and 'soft' endpoints on the relationship between risk factors and outcomes under the different statistical methods, several additional sensitivity analyses were performed: (1) using only non-fatal CV events; (2) including non-CV mortality in the combined endpoint; (3) using only 'hard' endpoints usually included in a composite endpoint (non-fatal MI, non-fatal stroke and CV mortality); (4) limited to 10-year follow-up; (5) limited to 5-year follow-up; (6) limited to patients included after 2007 to study a more contemporary patient population treated under the 2007 European Society of Cardiology guidelines or more recent guidelines $^{25}$; and (7) limited to non-smokers.

\section{Patient and public involvement}

Patients and/or the public were not involved in the design, or conduct, or reporting, or dissemination plans of this research.

\section{RESULTS \\ Baseline characteristics}

The characteristics of the 7239 patients with recent CVD at baseline included in the study population are shown in table 1. Compared with patients who had no recurrent CV event during follow-up, patients with one or more recurrent events generally had a less favourable risk profile at baseline (table 1). Compared with people who experienced multiple recurrent events during follow-up, those who experienced no recurrent events during follow-up were slightly younger, more often women, less often current smokers, have a lower SBP and more often used lipid-lowering drugs at baseline. They were on average recruited later in the study (37\% after 2011, compared with only $7 \%$ in patients who experienced more CV events).

\section{First and total events during follow-up}

During a median follow-up of 8.9 years (IQR 4.8-9.1), 1412 patients (19.5\%) had one CV event during follow-up and 1290 patients (17.8\%) had multiple CV events during follow-up (figure 1). During the course of the study, a total of 5457 recurrent CV events occurred, of which 2702 were first events within the study period (figure 2). The first recurrent event took place after a median of 3.2 years (IQR 0.9-7.1) from inclusion. There were an additional $2755 \mathrm{CV}$ events that occurred after the first event during the course of the cohort study. The second recurrent event took place after a median of 1.1 years (IQR 0.2-4.1) after the first recurrent event.

In comparison, there was a higher proportion of MALE and CV death among the additional events than among the first events $(24.2 \%$ vs $12.4 \%$ and $14.5 \%$ vs $11.7 \%$, respectively). In contrast, there were less non-fatal myocardial infarctions $(8.1 \%$ vs $11.8 \%)$, non-fatal strokes $(5.6 \%$ vs $9.4 \%)$, coronary revascularisation $(31.9 \%$ vs $35.5 \%)$ and other revascularisations $(15.7 \%$ vs $19.2 \%)$. Figure 3 shows the Kaplan-Meier curve and mean cumulative function plot for the first and total CV events, respectively.

\section{Relation between classic risk factors and total events}

Figure 4 shows the Kaplan-Meier curve and mean cumulative function plot for the first and total new CV events, respectively, stratified according to the presence of each risk 
Table 1 Baseline characteristics of study participants with no recurrent $C V$ event, a single recurrent $C V$ event or multiple recurrent $\mathrm{CV}$ events

\begin{tabular}{|c|c|c|c|}
\hline & No recurrent CV event & Single recurrent $\mathrm{CV}$ event & Multiple recurrent CV events \\
\hline & $\mathrm{n}=4537$ & $n=1412$ & $\mathrm{n}=1290$ \\
\hline Age (years) & $59 \pm 11$ & $61 \pm 10$ & $60 \pm 10$ \\
\hline Male sex & $3170(70)$ & $1088(77)$ & $989(77)$ \\
\hline Current smoking & $1271(28)$ & $473(33)$ & $529(41)$ \\
\hline Systolic blood pressure $(\mathrm{mm} \mathrm{Hg})$ & $137 \pm 20$ & $141 \pm 20$ & $143 \pm 22$ \\
\hline Diastolic blood pressure $(\mathrm{mm} \mathrm{Hg})$ & $81 \pm 11$ & $81 \pm 11$ & $81 \pm 11$ \\
\hline History of coronary artery disease & $2630(58)$ & $826(58)$ & $736(57)$ \\
\hline History of cerebrovascular disease & $1513(33)$ & $362(26)$ & $276(21)$ \\
\hline History of PAD or AAA & $1995(44)$ & $608(43)$ & $636(49)$ \\
\hline Type 2 diabetes & $577(13)$ & $246(17)$ & $256(20)$ \\
\hline Lipid-lowering medication & $3219(71)$ & $887(63)$ & $799(62)$ \\
\hline Blood pressure-lowering medication & $3332(73)$ & $1058(75)$ & $946(73)$ \\
\hline Antiplatelet medication & $3344(74)$ & $1053(75)$ & $907(70)$ \\
\hline Total cholesterol (mmol/L) & $4.7 \pm 1.2$ & $4.9 \pm 1.2$ & $5.1 \pm 1.3$ \\
\hline HDL cholesterol (mmol/L) & $1.3 \pm 0.4$ & $1.2 \pm 0.4$ & $1.2 \pm 0.3$ \\
\hline LDL cholesterol (mmol/L) & $2.7 \pm 1.0$ & $3.0 \pm 1.1$ & $3.2 \pm 1.1$ \\
\hline Serum creatinine (mmol/L; median) & $85(75-97)$ & $89(78-101)$ & $89(78-103)$ \\
\hline \multicolumn{4}{|l|}{ Inclusion year in UCC-SMART } \\
\hline 1996-2000 & $498(11)$ & $403(29)$ & 457 (35) \\
\hline 2001-2010 & $2373(52)$ & 829 (59) & $743(58)$ \\
\hline 2011-2018 & $1666(37)$ & $180(13)$ & $90(7)$ \\
\hline
\end{tabular}

Presented as $n(\%)$ for categorical data or mean $\pm S D$ for continuous data, unless stated otherwise.

AAA, abdominal aortic aneurysm; CV, cardiovascular; HDL, high-density lipoprotein; LDL, low-density lipoprotein; PAD, peripheral artery disease; UCC-SMART, Utrecht Cardiovascular Cohort - Second Manifestations of ARTerial disease.

factor. Table 2 shows the relationship between the classic risk factors of interest and (total) CV events as estimated by the different statistical models. Traditional time-to-first event Cox models showed significant relationships with first recurrent CV events for current smoking (HR 1.36, 95\% CI 1.25 to 1.48), non-HDL cholesterol (HR 1.14 per $\mathrm{mmol} / \mathrm{L}, 95 \%$

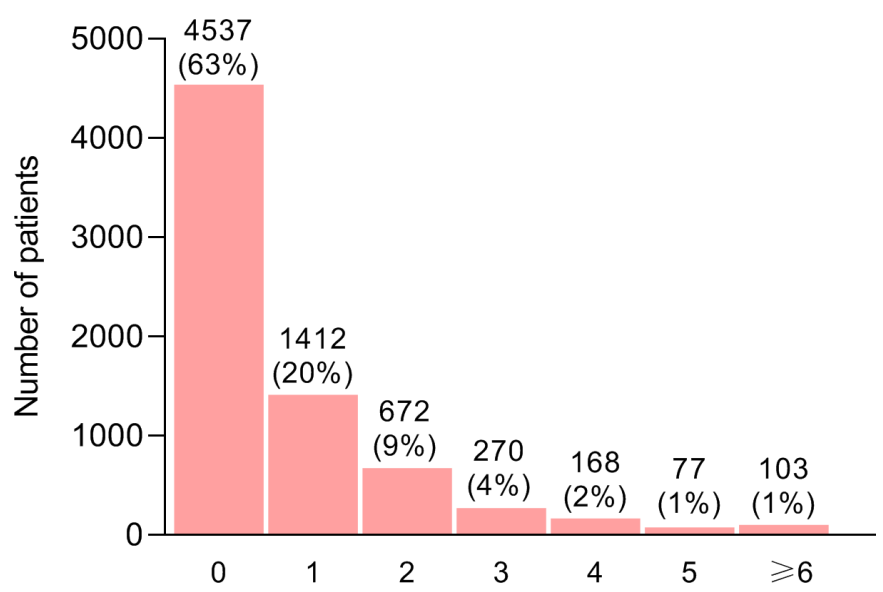

Cardiovascular events per patient during follow-up

Figure 1 Number of cardiovascular events per patient during follow-up.
CI 1.11 to 1.18 ) and SBP (HR 1.05 per $10 \mathrm{~mm} \mathrm{Hg}, 95 \%$ CI 1.03 to 1.07$)$. The PWP models showed significant relationship between the cumulative recurrent event burden and current smoking (HR 1.26, 95\% CI 1.17 to 1.35 ), non-HDL cholesterol (HR 1.09, 95\% CI 1.06 to 1.12) and SBP (HR $1.04,95 \%$ CI 1.03 to 1.06$)$.

\section{Sensitivity analyses}

Negative binomial regression and Andersen-Gill showed consistent results with regard to direction of the relation, but not in magnitude (table 2). For all risk factors, negative binomial regression showed stronger relationships than both Andersen-Gill and PWP. For all three risk factors, the relationship between event and risk factor was stronger for the fifth than for the first recurrent event, as shown by the WLW analysis. The results of the other sensitivity analyses are shown in table 3 . There were no important differences in the sensitivity analyses compared with the main analyses, with overlapping CIs.

\section{DISCUSSION}

In the current paper, we demonstrated that in patients with recently clinically manifest vascular disease, the modifiable risk factors current smoking, non-HDL cholesterol 


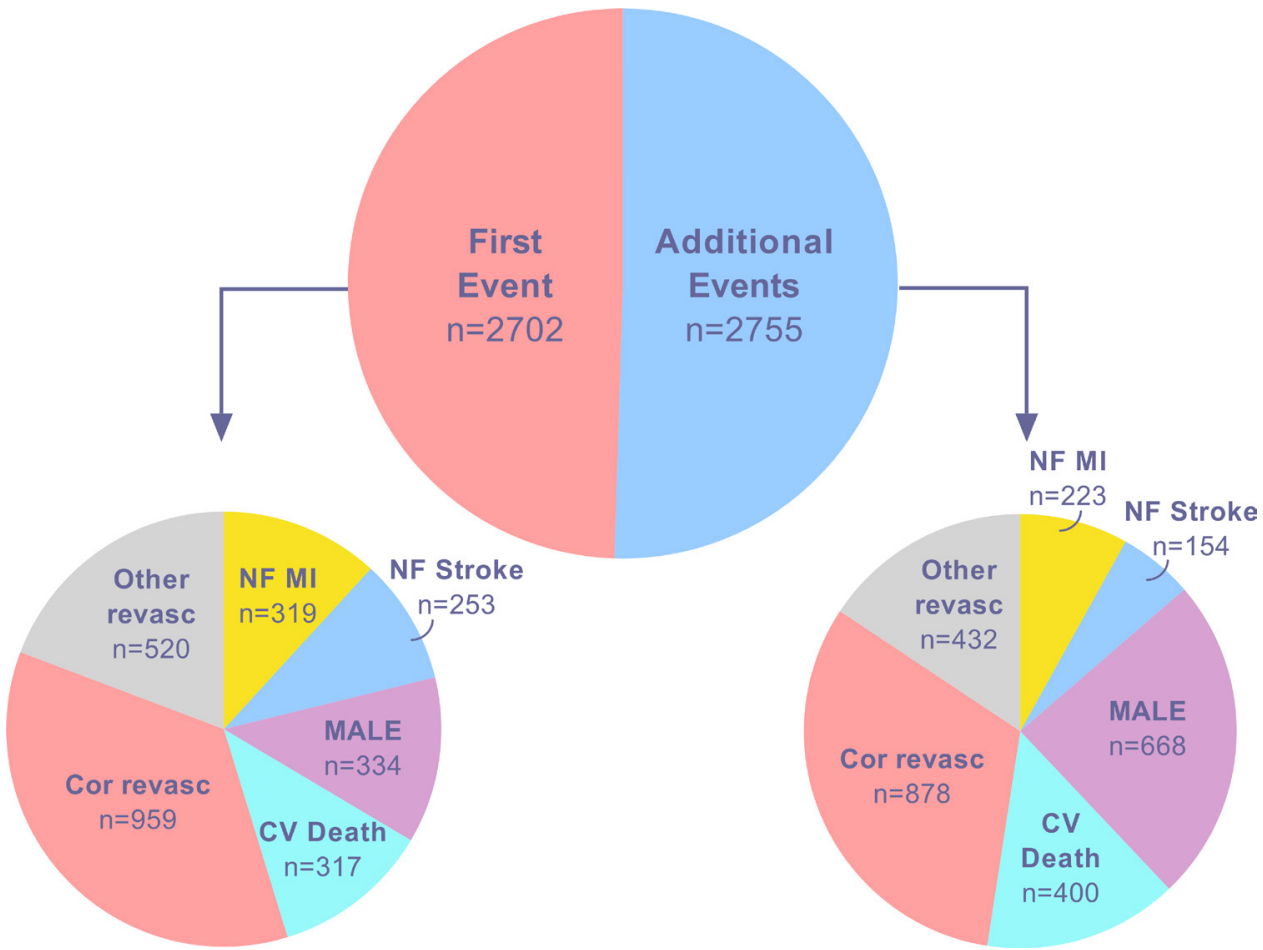

Figure 2 Number of first and total subsequent CV events and by individual endpoints. cor, coronary; CV, cardiovascular; MALE, major adverse limb events; MI, myocardial infarction; NF, non-fatal; revasc, revascularisation.

and SBP are not only related to the risk of a first recurrent $\mathrm{CV}$ event during follow-up, but also with total recurrent CV events over long-term follow-up.

Although not necessarily particularly surprising, the results of the present study confirm that better treatment of the classic risk factors is necessary in patients with clinically manifest vascular disease to prevent not just the first recurrent vascular event, but even more so the total burden of events. This is not only true for the hard (classic major adverse cardiovascular events (MACE))

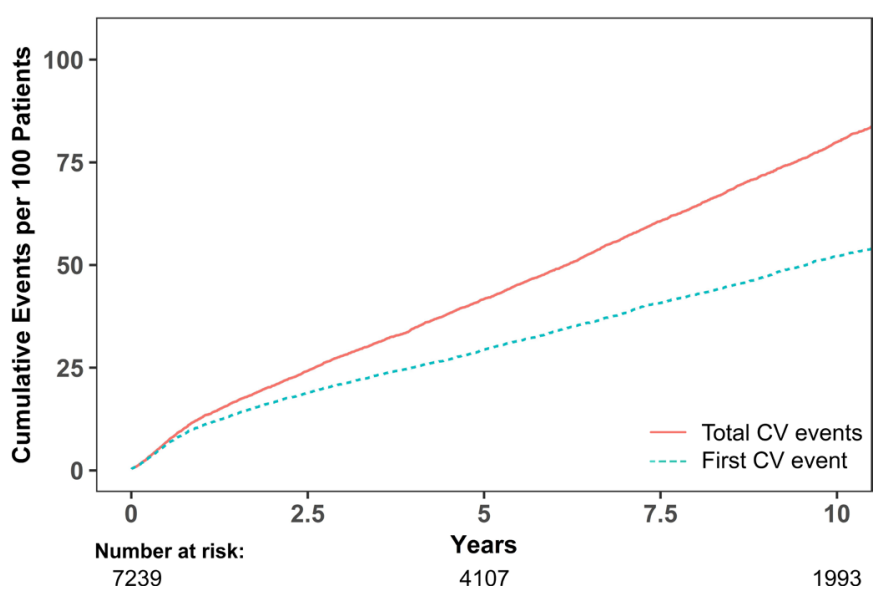

Figure 3 Mean cumulative functions and Kaplan-Meier curves for cardiovascular (CV) events. The mean cumulative function curve depicts the expected total number of recurrent CV events for a given 100 patients in the study population at a given time after randomisation. In contrast, the KaplanMeier curve depicts the expected number of patients with a first recurrent CV event at any given time per 100 patients. endpoints, but for all (non-fatal and fatal) endpoints. These results may be of use in communicating the benefit of risk factor treatment with patients in clinical practice. Although the effect sizes are smaller for the total recurrent events compared with only the first recurrent event, they are still clinically very important. Smoking at baseline is associated with a $26 \%$ increase in hazard for any recurrent event. As we have only used baseline smoking status in the current analysis, this does not account for people who quit smoking after baseline. Likely, the effect size of those patients who keep smoking throughout the study follow-up is larger. This is corroborated by previous analyses showing that smoking cessation importantly decreases the risk of future events. ${ }^{26}$ But the HRs for nonHDL cholesterol and SBP are also clinically important; a difference of $3 \mathrm{mmol} / \mathrm{L}$ in baseline non-HDL cholesterol is associated with a $30 \%$ increase in hazard for any recurrent event; a difference of $40 \mathrm{~mm} \mathrm{Hg}$ in baseline SBP is associated with a $17 \%$ increase in hazard.

Multiple events over time in a subject are associated with a large burden for the patient, decreased quality of life and higher costs. This is important not only at the patient level, but also for the healthcare systems as multiple events in a single patient lead to the use of more resources, due to hospitalisations, tests, additional (surgical) interventions and physician visits.

In recent years, several randomised controlled trials have published the effects of trial interventions on the total CV event burden. In the ODYSSEY OUTCOMES trial, which studied the addition of the proprotein convertase subtilisin/kexin type 9 (PCSK9) monoclonal 
(A)

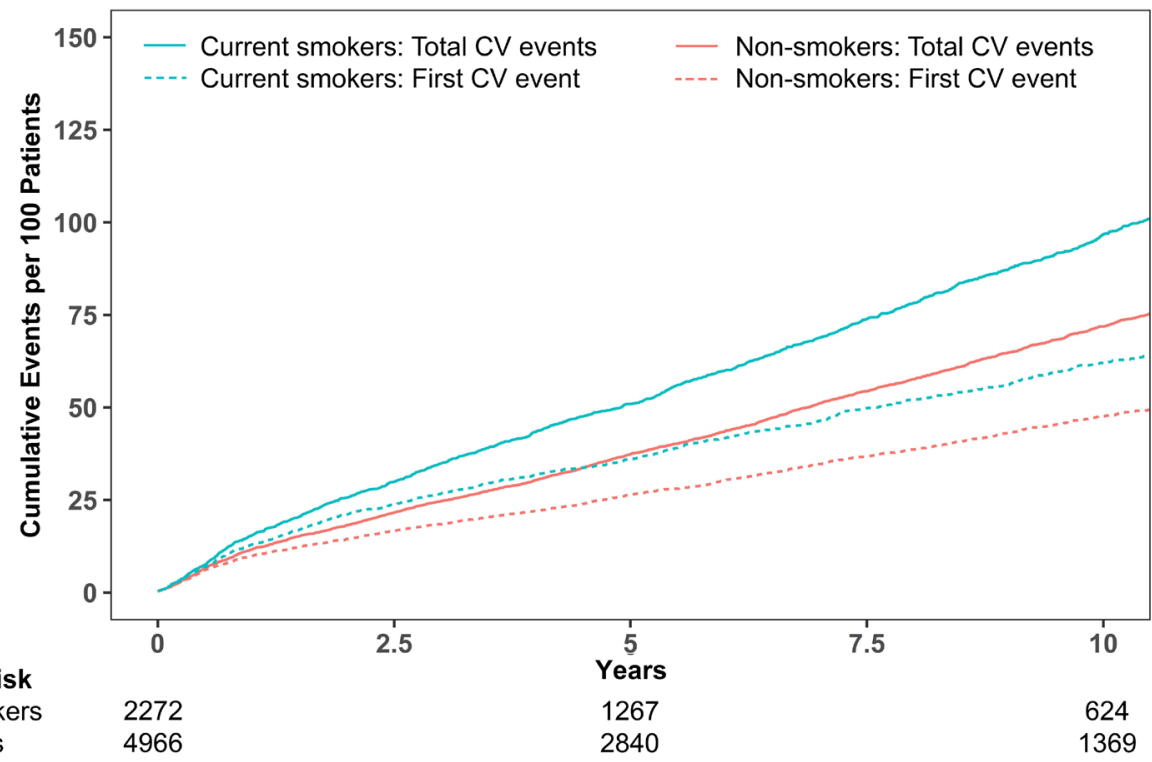

Number at risk

Current smokers 4966

1369

(B)

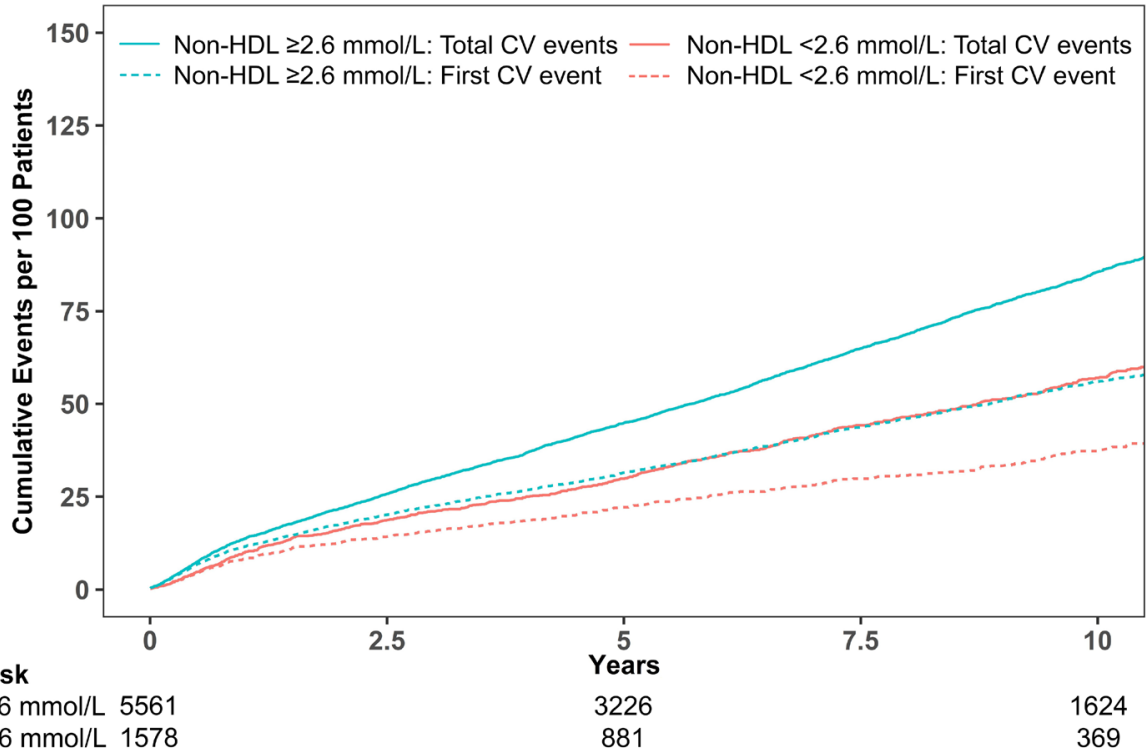

Non-HDL $\geq 2.6 \mathrm{mmol} / \mathrm{L} 5561$

Non-HDL $<2.6 \mathrm{mmol} / \mathrm{L} 1578$

369

(C)

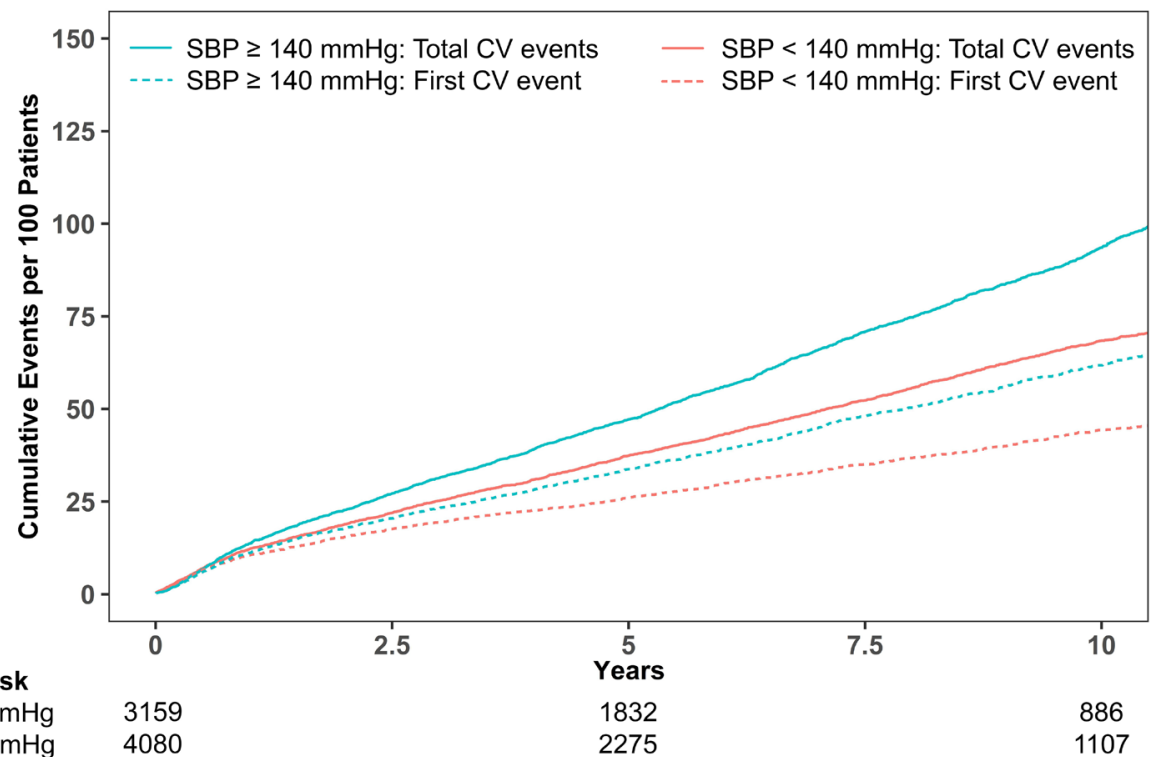

Figure 4 Mean cumulative functions and Kaplan-Meier curves for cardiovascular (CV) events, stratified according to (A) smoking status, (B) non-high-density lipoprotein (HDL) cholesterol and (C) systolic blood pressure (SBP). 
Table 2 Relation between traditional risk factors and first or total subsequent recurrent CV events according to different statistical methods

\begin{tabular}{|c|c|c|c|c|}
\hline & & Current smoking & $\begin{array}{l}\text { Non-HDL cholesterol } \\
\text { (per } \mathrm{mmol} / \mathrm{L} \text { ) }\end{array}$ & $\begin{array}{l}\text { SBP } \\
\text { (per } 10 \mathrm{~mm} \mathrm{Hg} \text { ) }\end{array}$ \\
\hline & Events* & HR $(95 \% \mathrm{Cl})$ & HR (95\% Cl) & HR (95\% Cl) \\
\hline Time-to-first event Cox regression & 2702 & $1.36(1.25$ to 1.48$)$ & 1.14 (1.11 to 1.18$)$ & 1.05 (1.03 to 1.07$)$ \\
\hline Prentice-Williams-Peterson & 5138 & 1.26 (1.17 to 1.35$)$ & 1.09 (1.06 to 1.12$)$ & 1.04 (1.03 to 1.06$)$ \\
\hline Negative binomial regression & 5138 & $1.33(1.22$ to 1.45$)$ & 1.23 (1.19 to 1.28$)$ & 1.07 (1.05 to 1.10$)$ \\
\hline Andersen-Gill & 5138 & 1.35 (1.24 to 1.47$)$ & 1.13 (1.09 to 1.16$)$ & 1.05 (1.04 to 1.07$)$ \\
\hline Wei-Lin-Weissfeld & 2702 & 1.41 (1.28 to 1.56$)$ & 1.15 (1.10 to 1.21$)$ & 1.06 (1.04 to 1.09$)$ \\
\hline \multicolumn{5}{|l|}{ Stratified per event } \\
\hline \multicolumn{5}{|l|}{ Prentice-Williams-Peterson } \\
\hline First event & 2702 & $1.36(1.25$ to 1.48$)$ & 1.14 (1.10 to 1.19$)$ & 1.05 (1.03 to 1.07$)$ \\
\hline Second event & 1290 & 1.17 (1.02 to 1.35$)$ & 1.09 (1.03 to 1.16$)$ & 1.03 (1.00 to 1.07 ) \\
\hline Third event & 618 & 1.04 (0.84 to 1.29$)$ & 0.99 (0.93 to 1.06$)$ & 1.09 (1.04 to 1.14$)$ \\
\hline Fourth event & 348 & 1.03 (0.77 to 1.38$)$ & 1.02 (0.95 to 1.08$)$ & 1.00 (0.94 to 1.06$)$ \\
\hline Fifth event & 180 & $1.40(0.90$ to 2.17$)$ & $1.03(0.93$ to 1.13$)$ & 1.03 (0.95 to 1.12$)$ \\
\hline \multicolumn{5}{|l|}{ Wei-Lin-Weissfeld } \\
\hline First event & 2702 & $1.36(1.25$ to 1.48$)$ & 1.14 (1.10 to 1.19$)$ & 1.05 (1.03 to 1.07$)$ \\
\hline Second event & 1290 & $1.49(1.32$ to 1.68$)$ & $1.18(1.12$ to 1.25$)$ & 1.06 (1.03 to 1.09$)$ \\
\hline Third event & 618 & 1.44 (1.21 to 1.72$)$ & 1.13 (1.05 to 1.22$)$ & 1.10 (1.06 to 1.14$)$ \\
\hline Fourth event & 348 & 1.43 (1.13 to 1.81$)$ & 1.13 (1.04 to 1.24$)$ & 1.12 (1.06 to 1.18$)$ \\
\hline Fifth event & 180 & 1.56 (1.12 to 2.17$)$ & $1.20(1.10$ to 1.31$)$ & 1.10 (1.02 to 1.18$)$ \\
\hline
\end{tabular}

*Limited to the first five recurrent events.

CV, cardiovascular; HDL, high-density lipoprotein; SBP, systolic blood pressure.

Table 3 Results of the sensitivity analyses

\begin{tabular}{|c|c|c|c|c|c|}
\hline \multirow[b]{2}{*}{ Scenario } & \multirow[b]{2}{*}{ Model } & \multirow[b]{2}{*}{ Events } & \multirow{2}{*}{$\begin{array}{l}\text { Current smoking } \\
\text { HR }(95 \% \mathrm{Cl})\end{array}$} & \multirow{2}{*}{$\begin{array}{l}\text { Non-HDL cholesterol } \\
\text { (per } \mathrm{mmol} / \mathrm{L})\end{array}$} & \multirow{2}{*}{$\begin{array}{l}\text { SBP } \\
\text { (per } 10 \text { mm Hg) } \\
\text { HR }(95 \% \mathrm{Cl})\end{array}$} \\
\hline & & & & & \\
\hline Main analysis & PWP & 5138 & 1.26 (1.17 to 1.35$)$ & 1.09 (1.06 to 1.12$)$ & 1.04 (1.03 to 1.06$)$ \\
\hline Only non-fatal events & Time-to-first event & 2385 & 1.34 (1.22 to 1.46$)$ & 1.15 (1.10 to 1.19$)$ & 1.05 (1.03 to 1.07$)$ \\
\hline $\begin{array}{l}\text { Non-fatal events and } \\
\text { all-cause mortality }\end{array}$ & PWP & 5940 & 1.33 (1.25 to 1.41$)$ & 1.08 (1.05 to 1.11$)$ & 1.04 (1.03 to 1.06$)$ \\
\hline \multirow{2}{*}{$\begin{array}{l}\text { Major cardiovascular } \\
\text { events }\end{array}$} & Time-to-first event & 1288 & 1.64 (1.45 to 1.85$)$ & 1.08 (1.03 to 1.14$)$ & 1.03 (1.01 to 1.06$)$ \\
\hline & PWP & 1660 & 1.52 (1.35 to 1.72$)$ & 1.07 (1.02 to 1.12$)$ & 1.04 (1.01 to 1.06$)$ \\
\hline $\begin{array}{l}\text { Limited to } 10 \text { years of } \\
\text { follow-up }\end{array}$ & Time-to-first event & 2368 & 1.39 (1.27 to 1.52$)$ & 1.14 (1.10 to 1.19$)$ & 1.05 (1.03 to 1.07$)$ \\
\hline \multirow{2}{*}{$\begin{array}{l}\text { Limited to patients } \\
\text { included after } 2007\end{array}$} & Time-to-first event & 644 & 1.40 (1.17 to 1.67$)$ & 1.12 (1.04 to 1.22$)$ & 1.04 (1.00 to 1.08$)$ \\
\hline & PWP & 1069 & 1.32 (1.15 to 1.53$)$ & 1.09 (1.02 to 1.18$)$ & 1.03 (0.99 to 1.06$)$ \\
\hline \multirow[t]{2}{*}{ Only never smokers } & Time-to-first event & 485 & N/A & 1.16 (1.06 to 1.27$)$ & $1.04(1.00$ to 1.09$)$ \\
\hline & PWP & 841 & $\mathrm{~N} / \mathrm{A}$ & 1.14 (1.06 to 1.23$)$ & $1.02(0.99$ to 1.06$)$ \\
\hline
\end{tabular}

HDL, high-density lipoprotein; N/A, not applicable; PWP, Prentice-Williams-Peterson; SBP, systolic blood pressure. 
antibody alirocumab to intensive statin therapy after acute coronary syndrome, non-fatal CV events were prevented in 162 patients during follow-up, while a total of 327 non-fatal CV events were prevented with alirocumab compared with placebo. ${ }^{7}$ Similarly, in the REDUCE-IT trial, icosapent ethyl prevented 196 first events compared with placebo, but 470 total events (from a composite of $\mathrm{CV}$ death, non-fatal myocardial infarction, non-fatal stroke, coronary revascularisation or hospitalisation for unstable angina) in patients with either established CVD or diabetes mellitus with additional risk factors. ${ }^{8}$ In the IMPROVE-IT trial, the addition of ezetimibe to simvastatin prevented 170 first events compared with placebo and 421 total events. ${ }^{5}$ In these trials, the efficacy of these therapies is thus amplified by studying the total event burden, giving a better reflection of the clinical impact in the respective patient populations.

In the current study, the total CV event burden is double the burden of the first CV events. Interestingly, the sensitivity analysis using the WLW method shows consistently for all three risk factors that the association between risk factor and the fifth event of a patient is larger than the association between risk factor and the first event of a patient, suggesting that these classic risk factors become more important for subsequent events. This is interesting as lipid-lowering and BP-lowering are cornerstones of secondary prevention in international guidelines. One would therefore expect that the relationship between lipid or BP levels at baseline and later recurrent events should be diminished. One hypothesis is that the group who has multiple recurrent events is most resistant to therapy. If part of the population meets the treatment targets, the effect of the risk factors in those who do not meet the treatment targets may be relatively more important. However, there may also be a statistical carry-over effect from the risk factor from event $k$ to event $k+1$, and it has been suggested that this statistical methodology may lead to overestimation of the effect. ${ }^{19}$ Finally, it should be realised that the WLW model was a sensitivity analysis. Nonetheless, it is an interesting finding that warrants attention, as it indicates that there are patients with important residual risk from modifiable risk factors, perhaps even suggesting that the preventive treatment may be inadequate in some patients under treatment in this tertiary centre.

The PWP strata-specific results give an opposite result from the WLW strata-specific results. Looking at the strataspecific coefficients using the PWP approach, however, it seems as though the association with the risk factors decreases between cumulative events. However, methodologically this is to be expected, as only patients who have had a $k-1$ and who are still at risk (ie, who have not died) are in the risk set for event $k$, and the risk of event $k-1$ was also associated with the risk factors of interest. Thus, this approach may give the impression that these risk factors become less important after more recurrent events, while this may very well be inherent to the statistical methodology.
As there are still many unanswered questions about total event analysis, we would not recommend replacing time-to-first event analyses with recurrent event analysis. However, due to the clinical impact of the total event burden compared with first events alone, we would recommend that recurrent event analysis should be used more often in observational research to study the impact of risk factors on the total disease burden.

The current study has several strengths. First, the total event burden is a metric that better captures the entire clinical impact of risk factors than first events only. Additionally, this gives an increased statistical power to study the effects of risk factors on outcomes. ${ }^{27}$ As the UCCSMART cohort has a long follow-up with a large number of CV events, this study is very suitable for studying these questions. Additional strengths include the prospective study design and the extensive and systematic availability of data on potential confounders. Finally, we performed several sensitivity analyses to confirm the validity of the results, including limiting the follow-up time to 10 and 5 years, as there may be up to 20 years of follow-up between baseline measurements and end of follow-up for some patients in the UCC-SMART cohort, which may potentially dilute the effect on recurrent events, especially since risk factor management was less stringent in the earlier years of the UCC-SMART cohort.

Several potential limitations of this study also need to be acknowledged. First, as mentioned before, only baseline measurements are studied. The current study therefore differs from the previously mentioned randomised controlled trials, where an intervention strategy that influences the risk factor of interest (ie, low-density lipoprotein cholesterol) is studied. Additionally, all endpoints are weighed equally in the endpoint, while in clinical practice not all endpoints have equal clinical relevance. A weighted effect measure, which has already been proposed in previous literature, could solve this issue. ${ }^{28}$ Such methods should be examined in future studies. Furthermore, the statistical methods for analysing subsequent events make certain assumptions regarding the relationship between subsequent events which may not be met. Therefore, to ascertain the validity of the results, we used several different statistical methodologies. Although the different statistical methods do not show consistency in magnitude of the relationship, there is consistency of findings in terms of direction. It has previously been demonstrated that the choice of statistical method for recurrent event analysis can impact the results and conclusion. ${ }^{29}{ }^{29}$ It is thus unclear which statistical method should be preferred for recurrent event analysis in observational CVD studies, although it has been suggested that PWP models may be the most robust option. ${ }^{2}$ Simulation studies have suggested that PWP should be the recommended approach for analysis of recurrent events in trials, ${ }^{30}$ although it is unclear whether this is also true for cohort studies. We therefore chose to use PWP as primary analyses and the other statistical methods as sensitivity analyses. Furthermore, the PWP models do not 
violate the assumption of proportionality. Finally, observational recurrent event analysis is at risk of index event bias, which generally tends to bias towards the null. ${ }^{31}$ This means that the HRs found in the current study may be underestimations of the true effect sizes.

In conclusion, in a cohort of patients with a recent first manifestation of established CVD, the total CV event burden during follow-up was twice as high as the number of first events. SBP, non-HDL cholesterol and current smoking are important modifiable risk factors for not only the first, but also for subsequent CV events, which are of great importance for patients in clinical practice. These results confirm the importance of the classic risk factors for total CV burden, underlining the necessity of optimal treatment of CV risk factors.

\section{Author affiliations}

${ }^{1}$ Department of Vascular Medicine, University Medical Centre Utrecht, Utrecht, The Netherlands

${ }^{2}$ UMC Utrecht, University Medical Centre Utrecht, Utrecht, The Netherlands

${ }^{3}$ Julius Center for Health Sciences and Primary Care, University Medical Center

Utrecht, Utrecht, The Netherlands

${ }^{4}$ Department of Cardiology, ICIN-Netherlands Heart Institute, Durrer Center for Cardiogenetic Research, University Medical Centre Utrecht, Utrecht, The Netherlands

${ }^{5}$ Institute of Cardiovascular Science, Faculty of Population Health Sciences, University College London, London, UK

${ }^{6}$ Department of Internal Medicine, Amsterdam UMC, Location VU University, Amsterdam, The Netherlands

${ }^{7}$ Department of Vascular Medicine, Utrecht University, Utrecht, The Netherlands

Acknowledgements The authors gratefully acknowledge the contribution of the SMART research nurses, $R$ van Petersen (data manager) and B Dinther (vascular manager), and the members of the Utrecht Cardiovascular Cohort - Second Manifestations of ARTerial disease study group (UCC-SMART study group): FW Asselbergs and HM Nathoe, Department of Cardiology; GJ de Borst, Department of Vascular Surgery; ML Bots and MI Geerlings, Julius Center for Health Sciences and Primary Care; MH Emmelot, Department of Geriatrics; PA de Jong and T Leiner, Department of Radiology; AT Lely, Department of Obstetrics and Gynecology; NP van der Kaaij, Department of Cardiothoracic Surgery; LJ Kappelle and YM Ruigrok, Department of Neurology; MC Verhaar, Department of Nephrology and Hypertension; and FLJ Visseren (chair) and J Westerink, Department of Vascular Medicine, University Medical Centre Utrecht and Utrecht University.

Contributors JW, MLB, FWA and FLJV were involved in the design, data collection and coordination of the SMART study as a whole, representing the SMART study team. The other members of the SMART study group read the manuscript and fully agreed with publication. TIdV conducted the statistical analysis and produced the first draft of the manuscript. TIdV, JW and FLJV contributed to the interpretation of data. JW, MLB, FWA, YMS and FLJV critically revised the manuscript. All authors reviewed the manuscript, revised it for important intellectual content and approved the final version.

Funding The SMART study was financially supported by a grant from the University Medical Centre Utrecht.

Competing interests FWA is supported by UCL Hospitals NIHR Biomedical Research Centre.

Patient consent for publication Not required.

Ethics approval The Medical Ethics Committee of the Utrecht University Medical Centre approved the study.

Provenance and peer review Not commissioned; externally peer reviewed.

Data availability statement Data are available upon reasonable request. Data used in this study may be accessed by contacting FLJV at F.L.J.Visseren@ umcutrecht.nl.

Open access This is an open access article distributed in accordance with the Creative Commons Attribution Non Commercial (CC BY-NC 4.0) license, which permits others to distribute, remix, adapt, build upon this work non-commercially, and license their derivative works on different terms, provided the original work is properly cited, appropriate credit is given, any changes made indicated, and the use is non-commercial. See: http://creativecommons.org/licenses/by-nc/4.0/.

\section{ORCID iDs}

Yvo M Smulders http://orcid.org/0000-0002-5904-0264

Frank L J Visseren http://orcid.org/0000-0003-3951-5223

\section{REFERENCES}

1 Anker SD, McMurray JJV. Time to move on from 'time-to-first': should all events be included in the analysis of clinical trials? Eur Heart J 2012;33:2764-5.

2 Yang W, Jepson C, Xie D, et al. Statistical methods for recurrent event analysis in cohort studies of CKD. Clin J Am Soc Nephrol 2017;12:2066-73.

3 Tikkanen MJ, Szarek M, Fayyad R, et al. Total cardiovascular disease burden: comparing intensive with moderate statin therapy insights from the ideal (incremental decrease in end points through aggressive lipid lowering) trial. J Am Coll Cardiol 2009;54:2353-7.

4 Murphy SA, Cannon CP, Wiviott SD, et al. Reduction in recurrent cardiovascular events with intensive lipid-lowering statin therapy compared with moderate lipid-lowering statin therapy after acute coronary syndromes from the prove IT-TIMI 22 (pravastatin or atorvastatin evaluation and infection Therapy-Thrombolysis in myocardial infarction 22) trial. J Am Coll Cardiol 2009;54:2358-62.

5 Murphy SA, Cannon CP, Blazing MA, et al. Reduction in total cardiovascular events with Ezetimibe/Simvastatin post-acute coronary syndrome: the IMPROVE-IT trial. J Am Coll Cardiol 2016;67:353-61.

6 LaRosa JC, Deedwania PC, Shepherd J, et al. Comparison of 80 versus $10 \mathrm{mg}$ of atorvastatin on occurrence of cardiovascular events after the first event (from the Treating to New Targets [TNT] trial). Am J Cardiol 2010;105:283-7.

7 Szarek M, White HD, Schwartz GG, et al. Alirocumab reduces total nonfatal cardiovascular and fatal events: the odyssey outcomes trial. J Am Coll Cardiol 2019;73:387-96.

8 Bhatt DL, Steg PG, Miller M, et al. Effects of Icosapent Ethyl on Total Ischemic Events: From REDUCE-IT. J Am Coll Cardiol 2019;73:2791-802.

9 Ip EH, Efendi A, Molenberghs G, et al. Comparison of risks of cardiovascular events in the elderly using standard survival analysis and multiple-events and recurrent-events methods. BMC Med Res Methodol 2015;15:15.

10 Chinwong S, Patumanond J, Chinwong D, et al. Reduction in total recurrent cardiovascular events in acute coronary syndrome patients with low-density lipoprotein cholesterol goal $<70 \mathrm{mg} / \mathrm{dL}$ : a real-life cohort in a developing country. Ther Clin Risk Manag 2016;12:353-60.

11 Engelen SE, van der Graaf Y, Stam-Slob MC, et al. Incidence of cardiovascular events and vascular interventions in patients with type 2 diabetes. Int J Cardiol 2017;248:301-7.

12 Simons PC, Algra A, van de Laak MF, et al. Second manifestations of arterial disease (smart) study: rationale and design. Eur J Epidemiol 1999;15:773-81.

13 Donders ART, van der Heijden GJMG, Stijnen T, et al. Review: a gentle introduction to imputation of missing values. $J$ Clin Epidemiol 2006;59:1087-91.

14 Aalen $\mathrm{O}$. Nonparametric inference for a family of counting processes. Ann Stat 1978;6:701-26.

15 Piepoli MF, Hoes AW, Agewall S. Task force members. 2016 European guidelines on cardiovascular disease prevention in clinical practice: the sixth joint Task force of the European Society of cardiology and other societies on cardiovascular disease prevention in clinical practice. Eur Heart $J$ 2016;37:2315-81.

16 Prentice RL, Williams BJ, Peterson AV. On the regression analysis of multivariate failure time data. Biometrika 1981;68:373-9.

17 Rauch G, Kieser M, Binder H, et al. Time-to-first-event versus recurrent-event analysis: points to consider for selecting a meaningful analysis strategy in clinical trials with composite endpoints. Clin Res Cardiol 2018;107:437-43.

18 Rogers JK, Pocock SJ, McMurray JJV, et al. Analysing recurrent hospitalizations in heart failure: a review of statistical methodology, with application to CHARM-Preserved. Eur J Heart Fail 2014;16:33-40.

19 Rogers JK, Yaroshinsky A, Pocock SJ, et al. Analysis of recurrent events with an associated informative dropout time: application of the joint frailty model. Stat Med 2016;35:2195-205. 
20 Glynn RJ, Buring JE. Ways of measuring rates of recurrent events. BMJ 1996;312:364-7.

21 Jahn-Eimermacher A. Comparison of the Andersen-Gill model with Poisson and negative binomial regression on recurrent event data. Comput Stat Data Anal 2008;52:4989-97.

22 Andersen PK, Gill RD. Cox's Regression Model for Counting Processes: A Large Sample Study. Ann Stat 1982;10:1100-20.

23 Wei LJ, Lin DY, Weissfeld L. Regression analysis of multivariate incomplete failure time data by modeling marginal distributions. J Am Stat Assoc 1989;84:1065-73.

24 Lin DY, Wei LJ. The robust inference for the COX proportional hazards model. J Am Stat Assoc 1989;84:1074-8.

25 Graham I, Atar D, Borch-Johnsen K, et al. European guidelines on cardiovascular disease prevention in clinical practice: Executive summary: fourth joint Task force of the European Society of cardiology and other societies on cardiovascular disease prevention in clinical practice (constituted by representatives of nine societies and by invited experts). Eur Heart J 2007;28:2375-414.

26 van den Berg MJ, van der Graaf Y, Deckers JW, et al. Smoking cessation and risk of recurrent cardiovascular events and mortality after a first manifestation of arterial disease. Am Heart $J$ 2019;213:112-22 http://www.ncbi.nlm.nih.gov/pubmed/31132583

27 Rogers JK, Jhund PS, Perez A-C, et al. Effect of rosuvastatin on repeat heart failure hospitalizations: the corona trial (controlled rosuvastatin multinational trial in heart failure). JACC Heart Fail 2014;2:289-97.

28 Rauch G, Kunzmann K, Kieser M, et al. A weighted combined effect measure for the analysis of a composite time-to-first-event endpoint with components of different clinical relevance. Stat Med 2018;37:749-67.

29 Bakal JA, Roe MT, Ohman EM, et al. Applying novel methods to assess clinical outcomes: insights from the trilogy ACS trial. Eur Heart J 2015;36:385-92.

30 Ozga A-K, Kieser M, Rauch G. A systematic comparison of recurrent event models for application to composite endpoints. BMC Med Res Methodol 2018;18:2.

31 Dahabreh IJ, Kent DM. Index event bias as an explanation for the paradoxes of recurrence risk research. JAMA 2011;305:822-3. 